\title{
BMJ Open Predictors of HIV virological failure and drug resistance in Chinese patients after 48 months of antiretroviral treatment, 2008-2012: a prospective cohort study
}

\author{
Wei Kan, ${ }^{1}$ Tao Teng, ${ }^{1}$ Shujia Liang, ${ }^{2}$ Yanling Ma, ${ }^{3}$ Heng Tang, ${ }^{4}$ Tuerdi Zuohela, ${ }^{5}$ \\ Guoqing Sun, ${ }^{6}$ Cui He, ${ }^{1}$ Kristin M Wall, ${ }^{7}$ Vincent C Marconi, ${ }^{8,9}$ Lingjie Liao, ${ }^{1}$ \\ Xuebing Leng, ${ }^{1}$ Pengtao Liu, ${ }^{1}$ Yuhua Ruan, ${ }^{1,10}$ Hui Xing, ${ }^{1}$ Yiming Shao ${ }^{1}$
}

To cite: Kan W, Teng T, Liang S, et al. Predictors of HIV virological failure and drug resistance in Chinese patients after 48 months of antiretroviral treatment, 2008-2012: a prospective cohort study. BMJ Open 2017;7:e016012. doi:10.1136/ bmjopen-2017-016012

- Prepublication history for this paper is available online. To view these, files please visit the journal online (http://dx.doi org/10.1136/bmjopen-2017016012).

WK and TT contributed equally.

Received 21 January 2017

Revised 24 July 2017

Accepted 25 July 2017

CrossMark

For numbered affiliations see end of article.

Correspondence to

Dr Yiming Shao;

ysha008@gmail.com

\section{ABSTRACT}

Objective To explore factors associated with HIV virological failure (VF) and HIV drug resistance (HIVDR) among HIV-positive Chinese individuals 4 years after initiating first-line lamivudine-based antiretroviral treatment (ART) in 2008 at five sentinel sites.

Design First-line ART initiators who were previously treatment naïve were selected using consecutive ID numbers from the 2008 National Surveillance Database into a prospective cohort study. Questionnaires and blood samples were collected in 2011 and 2012 to assess the outcomes of interest: VF (defined as viral load $\geq 1000$ copies/mL) and HIVDR (defined as VF with genetic drug-resistant mutations). Questionnaires and data from National Surveillance Database assessed demographics and drug adherence data.

Results 536 individuals with HIV were analysed; the 4-year risk of VF was 63 (11.8\%) and HIVDR was 27 (5.0\%). Female participants initiating stavudine (D4T)based regimens were more susceptible to both VF (adjusted OR $(\mathrm{aOR})=2.5,95 \% \mathrm{Cl} 1$ to $6.1, \mathrm{p}=0.04)$ and HIVDR ( $\mathrm{aOR}=3.6,95 \% \mathrm{Cl} 1$ to $12.6, \mathrm{p}=0.05$ ) versus zidovudine-based regimens. Male participants missing doses in past month were more susceptible to both VF (aOR=2.8, 95\% $\mathrm{Cl} 1.1$ to $7, \mathrm{p}=0.03$ ) and HIVDR (aOR=9.7, $95 \% \mathrm{Cl} 2.1$ to $44.1, \mathrm{p}<0.01)$. Participants of non-Han nationality were of increased risk for HIVDR (aOR from 4.8 to $12.2, p<0.05$ ) and non-Han men were at increased risk for $\mathrm{VF}(\mathrm{aOR}=2.9,95 \% \mathrm{Cl} 1.1$ to $7.3, \mathrm{p}=0.02)$. All 27 participants detected with HIVDR had non-nucleoside reverse-transcriptase inhibitor mutations, 21 (77.8\%) also had nucleoside reverse-transcriptase inhibitor mutations, and no protease inhibitor mutations were detected.

Conclusions Our findings suggest successful treatment outcomes at 4 years for roughly $90 \%$ of patients. We suggest conducting further study on whether and when to change ART regimen for women initiated with D4T-based regimen, and reinforcing adherence counselling for men. Increased VF and HIVDR risk among non-Han minorities warrants further exploration, and ethnic minorities may be an important group to tailor adherence-focused interventions.
Strengths and limitations of this study

- We studied 48-month risk of virological failure (VF) and HIV drug resistance (HIVDR) and their associations with demographic and behavioural information among individuals across five sentinel sites.

- Study found drug adherence and adverse effects influenced VF and HIVDR differently across gender.

- The outcomes were measured in 2011 and 2012, and thus we may be missing transient VF outcomes.

\section{INTRODUCTION}

Antiretroviral treatment (ART) has dramatically improved health outcomes and decreased HIV-associated morbidity and mortality through virological suppression and subsequent CD4 recovery. ${ }^{1-4}$ In 2003, China launched a National Free Antiretroviral Treatment Program (NFATP) that includes lifelong provision of free ART for people living with HIV who met the national treatment criteria. ${ }^{56}$ The national treatment criteria from 2008 to 2011 were: (1) CD4 cell count $\leq 0.2 \times 10^{\wedge} 9 / \mathrm{L}$; (2) WHO stage III $/ \mathrm{IV}$ diseases; or (3) willingness to receive ART, regardless of criteria 1 and $2 .^{7}$

The State Council AIDS Working Committee Office and the United Nations Theme Group on AIDS estimated that there were more than 700000 persons living with HIV in China in 2008, and more than 52000 individuals with HIV across 31 provinces, autonomous regions and municipalities had received ART (made freely available by the NFATP) by August $2008 .^{8}$

With the rapid scale-up of treatment and challenges with adherence, virological failure (VF) and HIV drug resistance (HIVDR) are ever present and mounting concerns. Incomplete virological suppression, a major cause 
of HIVDR, compromises therapeutic efficacy for the individual receiving treatment, increasing the risk of viral rebound and opportunistic infections, and increases the risk of transmitting drug-resistant strains to other individuals in the general population..$^{9-11}$

Observational studies in China have documented the prevalence of VF and HIVDR strains among treated individuals living with HIV. A cross-sectional study conducted in Yunnan, Guangxi and Xinjiang provinces in 2010 stated that 1-year HIVDR prevalence was $4.1 \% .{ }^{5}$ VF prevalence for sexual transmitted population and intravenous drug users (IDUs) was $8.3 \%$ and $19.3 \%$, separately. A 6-year follow-up study in 2010 suggested an incidence of 14.1 per 100 person-years for $\mathrm{VF}$ and 11.9 per 100 personyears for HIVDR among former plasma donors in Anhui Province. $^{12}$

NFATP recommended to switch the first-line regimen from didanosine to lamivudine (3TC) in 2008, and there are few nationwide, prospective studies in China reporting frequency or predictors of VF and HIVDR for people after initiating 3TC-based regimens.

The aim of this study is to evaluate predictors of $\mathrm{VF}$ and HIVDR in a prospective cohort of Chinese HIV individuals with HIV 4 years after first initiating first-line 3TC-based ART in 2008 at five sentinel sites. We stratified our analyses by gender based on conflicting findings on gender differences both in virological responses and drug resistance to different ART regimen, as well as gender differences in ART adherence. ${ }^{13-16}$ To our knowledge, this is the first long-term study to evaluate VF and HIVDR on 3TC-based regimens.

\section{ETHICS STATEMENT}

The study was approved by the Institutional Review Board of the National Center for AIDS/STD Control and Prevention of the Chinese Center for Disease Control and Prevention (NCAIDS, China CDC). All participants provided written informed consent before participation. Signed informed consent was obtained from each of the participants prior to the interviews and blood collection.

\section{METHODS}

\section{Study design and data collection}

This study was designed under the WHO surveillance of HIVDR in adults receiving ART for 48 months. ${ }^{10} 1718$ Five provinces in China with highest rate of transmission were selected to conduct a prospective cohort study with a follow-up study at 12 months: Guangxi, Henan, Hubei, Xinjiang and Yunnan. Participants were sampled from the 2008 National HIV Surveillance Database through sequential sampling from each province. Participant eligibility criteria included being aged $\geq 18$ years; having initiated NFATP-sponsored first-line ART in 2008; having been ART free before 2008; having been on ART for $36 \pm 6$ months in 2011; and providing consent to participate in the study.
Questionnaires administered by trained study personnel from provincial CDC in private rooms using structured interviews collected data in 2011 and 2012. Each study participant was assigned a confidential identification number used to label questionnaire and blood specimen. Additional HIV-specific data including route of transmission, initial ART regimen, latest ART regimen, ART distribution location and CD4 cell count were collected from the 2011-2012 National HIV Surveillance Database. There were no missing demographic data, missed questionnaire data were fed back to local CDC for recollection at the time.

\section{Laboratory analysis}

Blood specimens were collected from all participants to determine CD4 cell count, HIV-1 RNA viral load (VL) and HIV-1 drug resistance mutations in both 2011 and 2012. CD4 cell count estimation was conducted at provincial CDC laboratories using flow cytometry (FACSCalibur, BD, USA) within 24 hours after specimen collection. Plasma was isolated and stored at $-80^{\circ} \mathrm{C}$ at a provincial CDC laboratory and then transferred to NCAIDS for testing HIV VL and drug mutation. Plasma HIV RNA was quantified with real-time NASBA (NucliSENS EasyQ bioMerieux, France) or COBAS (Roche Applied Biosystems, Germany) according to manufacturer's recommendations using in-house PCR, both of the assays were performed automatically. ${ }^{19} \mathrm{VF}$ was defined as VL $\geq 1000$ copies $/ \mathrm{mL}$. According to WHO protocol, ${ }^{20}$ HIVDR tests were performed on samples with VL $\geq 1000$ copies/mL. HIV-1 pol gene (protease 1-99 amino acids and part of reverse-transcriptase 1-252 amino acids) was amplified, purified and analysed using the Stanford HIV Drug Resistance Database (https://hivdb.stanford.edu/hivdb). Levels of HIVDR were classified according to the algorithm of Stanford HIVdb program. The scores are the sum of each mutation penalty score for a drug. Scores less than 10 indicate susceptible; scores between 10 and 14 indicate potential low-level resistance; scores between 15 and 29 indicate low-level resistance; scores between 30 and 59 indicate intermediate resistance. Scores of 60 or greater indicate high-level resistance. Any low, intermediate or high-level resistance identified was defined as HIVDR. ${ }^{21-24}$

\section{Data analysis}

Questionnaire data were double entered using EpiData V.3.1 (The EpiData Association, Odense, Denmark). Statistical Analysis System (SAS V.9.4, SAS Institute) was then used for data cleaning and analyses.

A 48-month risk for the outcomes of interest was calculated as the proportion of unique persons who had experienced incident VF or HIVDR by the end of follow-up in 2012.

Covariates of interest were described using counts and percentages overall and by the outcome of interest, stratified by gender. Univariate logistic regression models were constructed to explore associations between covariates of interest and VF or HIVDR. OR and 95\% CIs are reported. 
Variables that were significant $(\mathrm{p}<0.05)$ in the univariate models were then fit into multivariate logistic regression models assessment for removal of collinear variables that had the weakest association with the outcome. Adjusted ORs (aOR) and 95\% CIs were presented. $\mathrm{p}<0.05$ was defined as statistically significant, and all tests were two sided. Descriptive analysis on HIVDR mutation results was conducted among 27 HIVDR participants, stratified by sex.

\section{RESULTS}

Up to 1100 subjects were selected using consecutive ID numbers from 2008 National Surveillance Database; of those, 490 were lost to follow-up by December 2012. Among those lost to follow-up, 139 died, 55 emigrated, 134 lost contact, 17 refused to participate, 65 stopped ART before 30 months, 36 transferred, 8 were under custody, 6 failed to provide a blood sample, 3 switched from ART to Tangcao tablet (an antiviral Chinese herbal therapy), 2 became pregnant and switched to other regimens and 1 was paralysed. After excluding 74 participants for failing the eligibility criteria, 536 participants were included in the final 24-month analysis (figure 1). The 48-month risk of $\mathrm{VF}$ was $11.8 \%$ and risk of drug resistance was $5 \%$.

\section{Demographic and ART information (tables 1 and 2)}

Of the 536 eligible participants, $51.8 \%$ were male, $76.5 \%$ were of Han nationality, $45.0 \%$ had an education level of elementary school or less, $56.2 \%$ were farmers and $10.6 \%$ were unemployed with the rest having regular income.

All regimens in this cohort remained 3TC based from 2008 to 2012. Initiated nucleoside reverse-transcriptase inhibitor (NRTI) regimens included zidovudine (AZT) $(\mathrm{n}=349,65.1 \%)$ or stavudine (D4T) $(\mathrm{n}=187$, $34.9 \%)$. Sixty-six $(12.3 \%)$ participants later changed to tenofovir (TDF)-based regimen. Initiated non-nucleoside reverse-transcriptase inhibitor (NNRTI) regimens

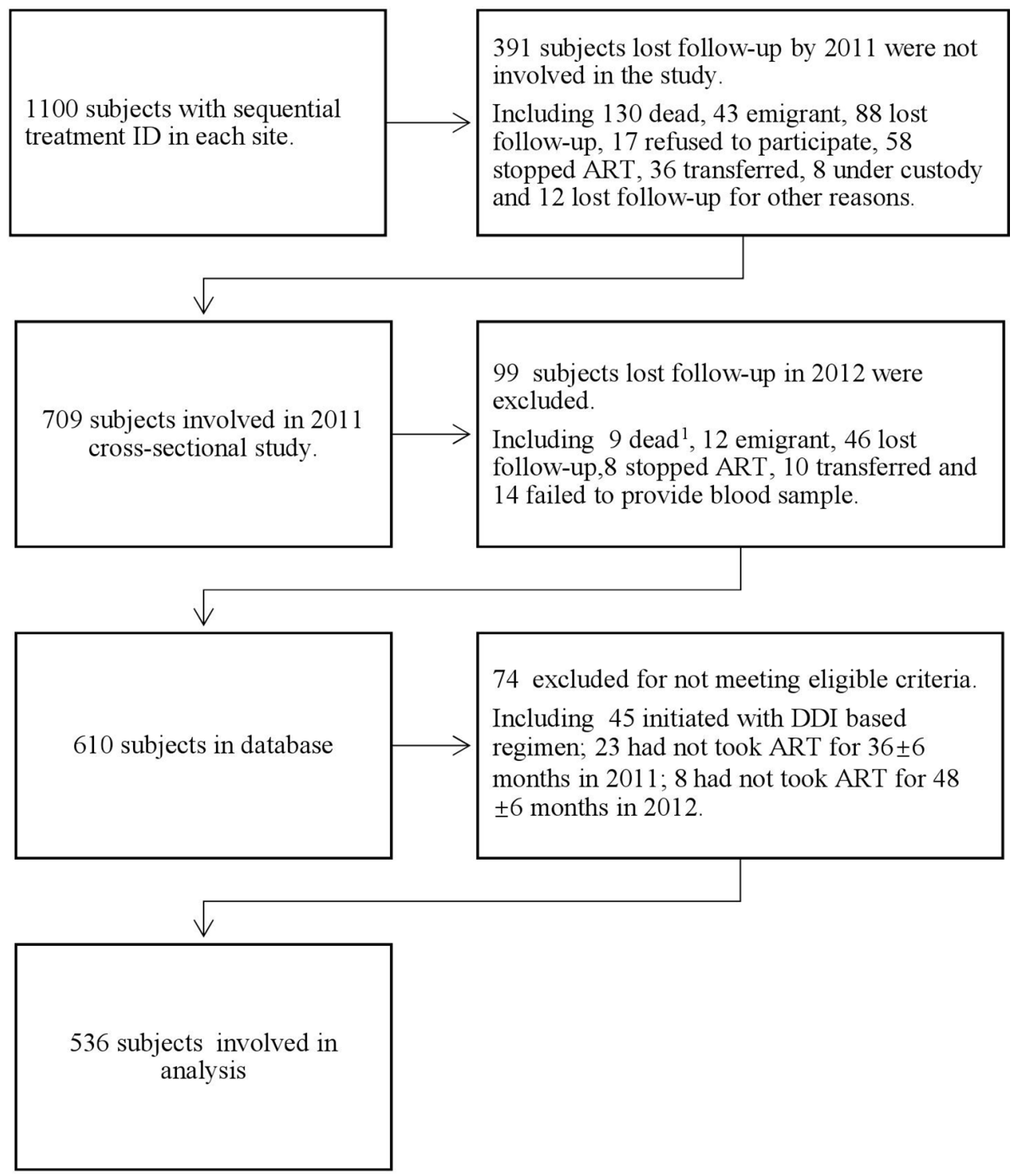

Figure 1 Figure of exclusion on study cohort. ${ }^{1}$ None HIV-related death. ART, antiretroviral treatment; DDI, didanosine. 


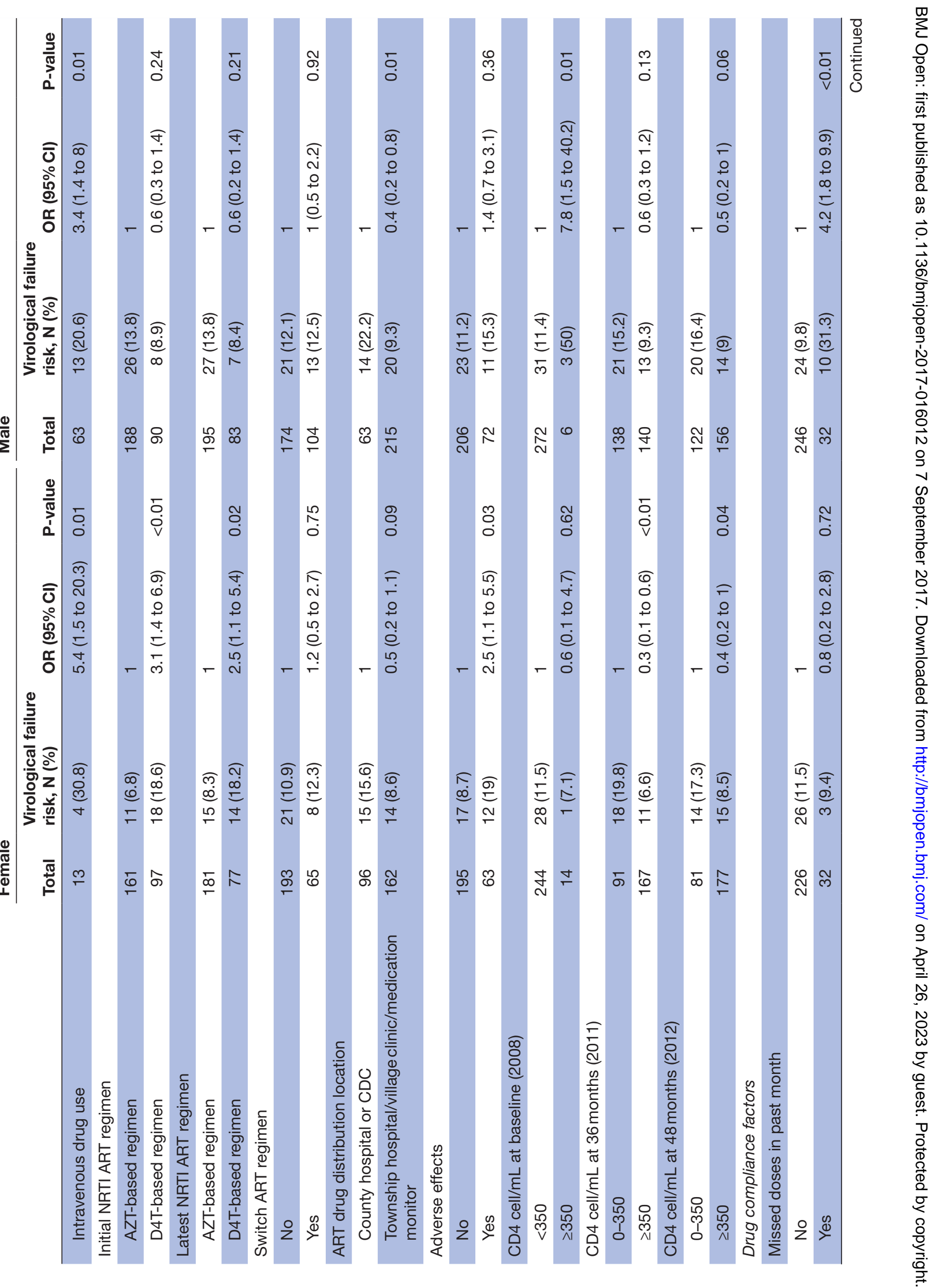




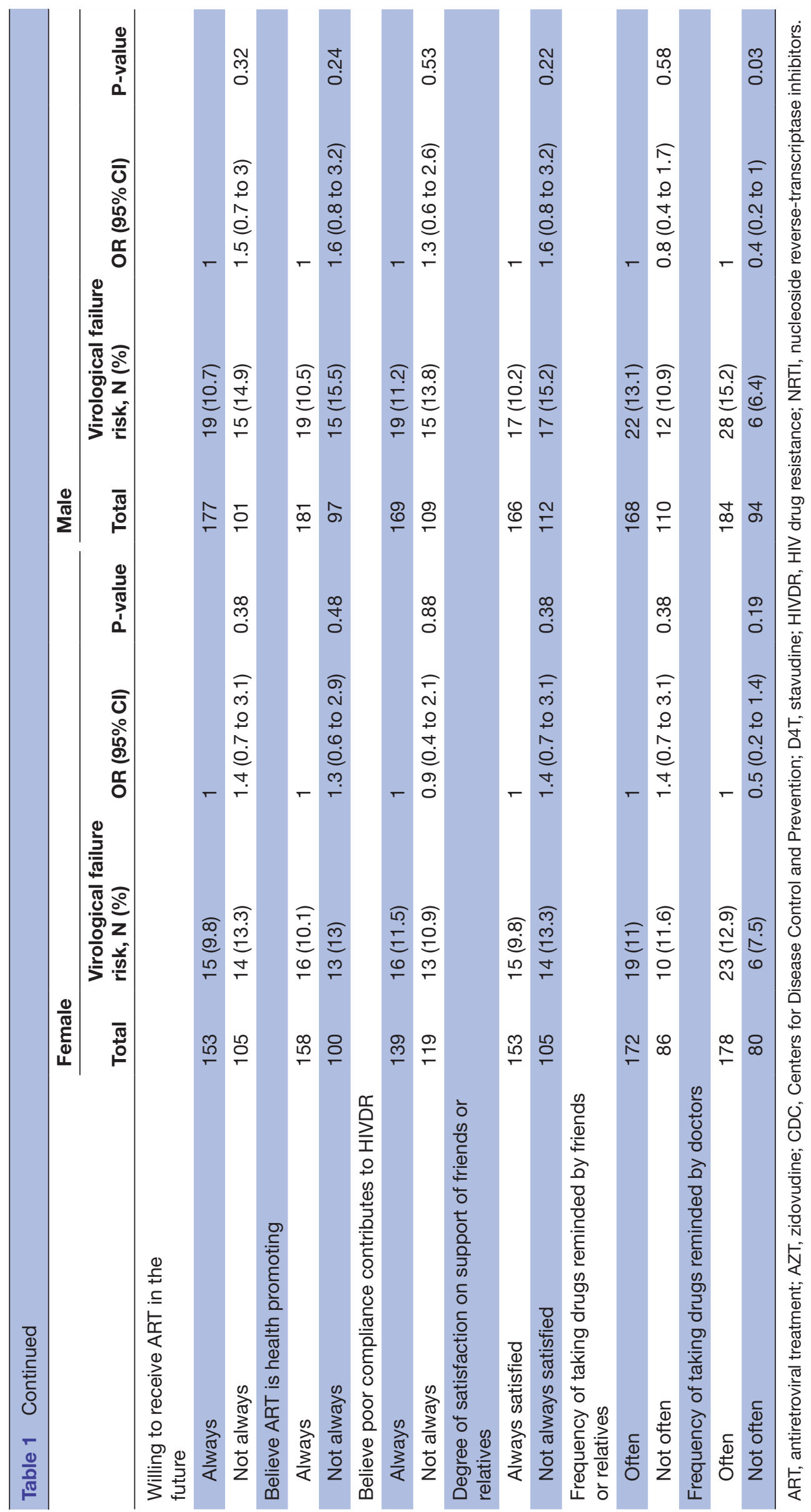




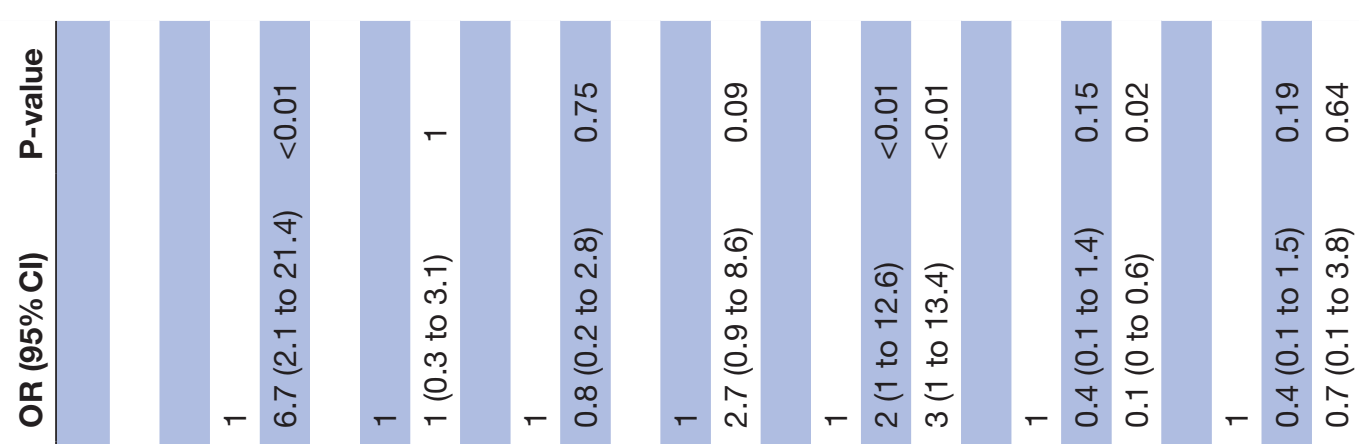

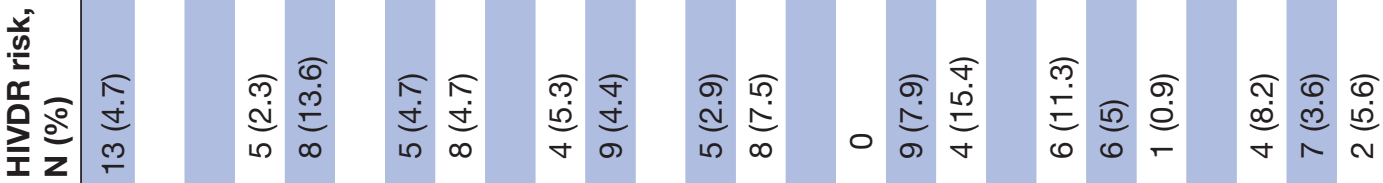

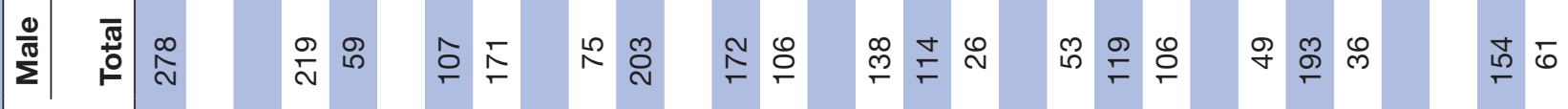

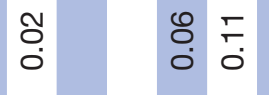

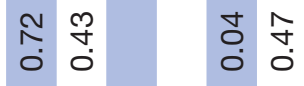

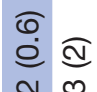

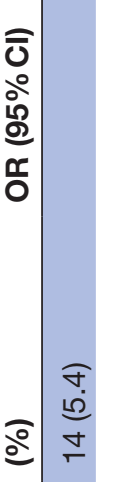

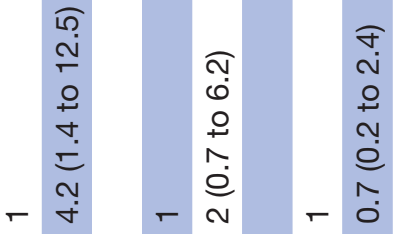

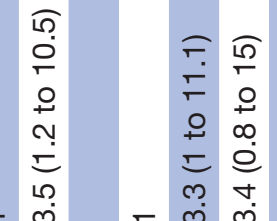

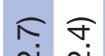

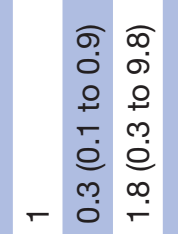

๑)

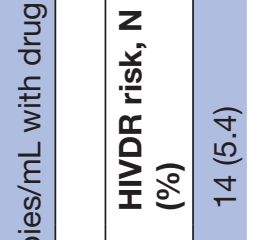

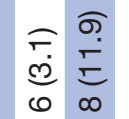

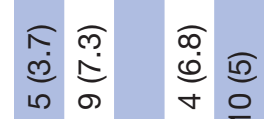

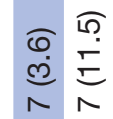

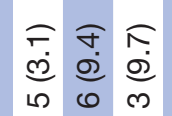

$\begin{array}{lll}\widehat{0} & \widehat{\sigma} & \sigma \\ \dot{\theta} & \dot{\sigma} & \dot{m}\end{array}$

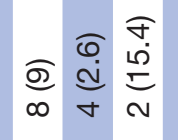

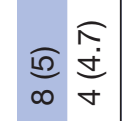

ธุ

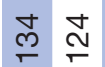

요 음

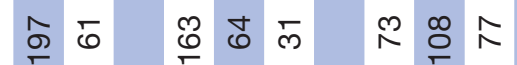

வ

온 $\infty$

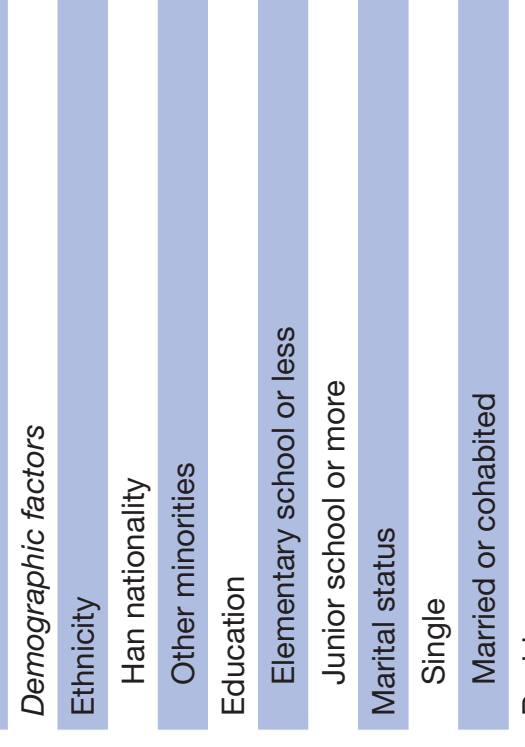

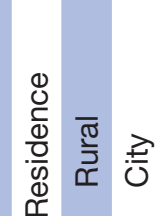




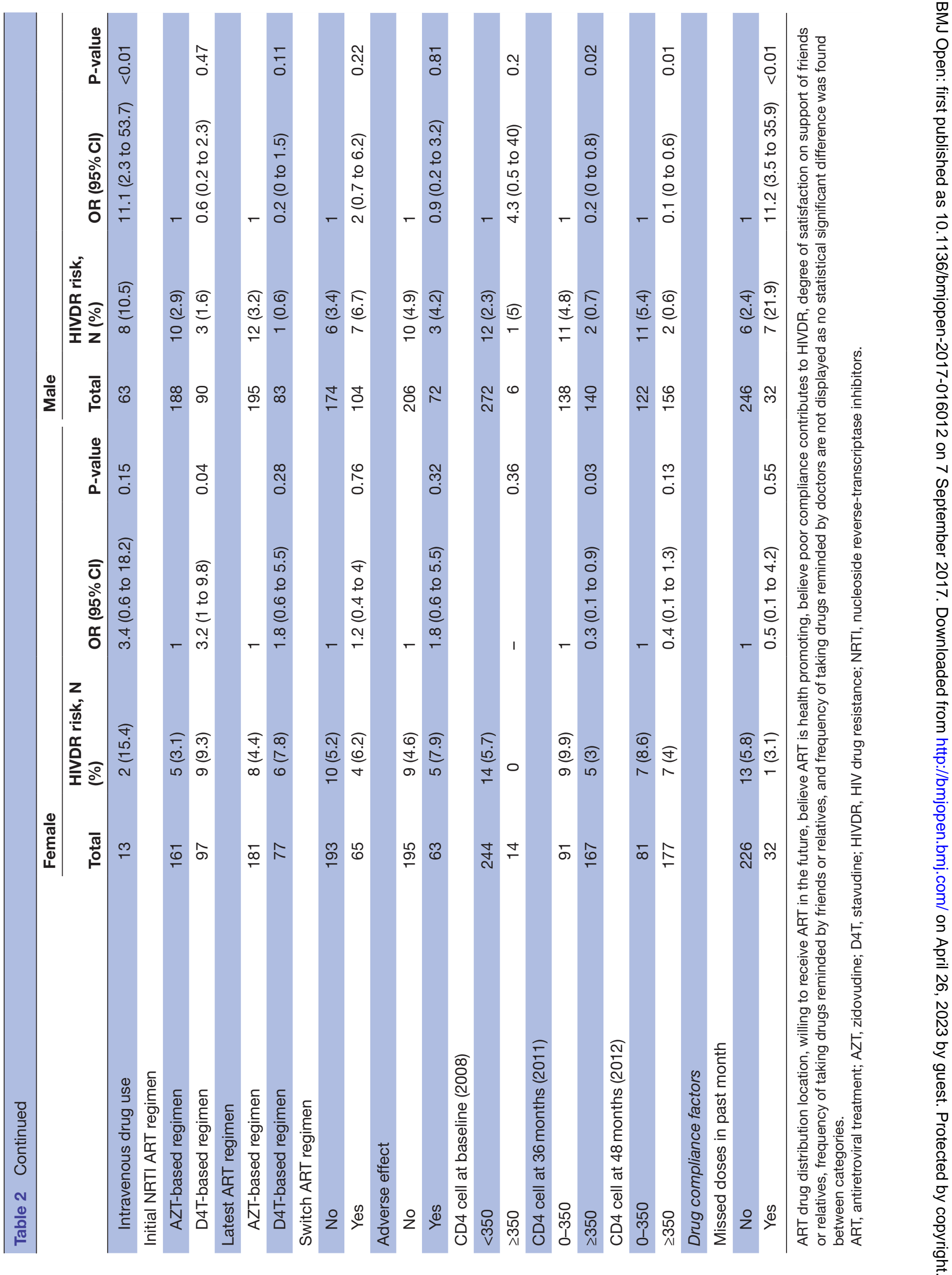


included nevirapine $(n=421,78.5 \%)$ or etravirine $(n=115$, $21.5 \%)$. Fifty-five $(10.3 \%)$ participants later changed to $\mathrm{LPV} / \mathrm{r}$ (Lopinavir/Ritonavir)-based regimen. One hundred and sixty-nine $(31.5 \%)$ participants switched the initial ART regimen during 2008-2012, but no statistical significant difference was found in VF $(p=0.74)$ and HIVDR ( $p=0.29$ ) risk between participants who switched regimens and participants who did not switch regimens.

We found that $38.4 \%$ of participants were hesitant to accept ART in the future, $36.8 \%$ participants reported doubts whether ART was health promoting and 42.5\% participants did not report that poor ART adherence necessarily contributed to HIVDR. Additionally, 40.5\% of participants were not always satisfied with support from friends or relatives. Four hundred and seventy-two $(88.1 \%)$ participants reported not missing a dose in the month prior to the date of the survey.

\section{Multivariate model results (table 3)}

As shown in table 3, minority male participants were at higher risk for both VF (aOR=2.9, 95\% CI 1.1 to 7.3, $\mathrm{p}=0.02$ ) and HIVDR (aOR=12.2, 95\% CI 1.8 to 84.8 , $\mathrm{p}=0.01$ ) compared with Han nationality male participants, while female minorities were only at a higher risk for HIVDR $(\mathrm{aOR}=4.8,95 \%$ CI 1.2 to 19.7, $\mathrm{p}=0.03)$.

Female participants initiating D4T-based regimens were at a higher risk for both $\mathrm{VF}(\mathrm{aOR}=2.5,95 \% \mathrm{CI} 1$ to 6.1 , $\mathrm{p}=0.04)$ and HIVDR ( $\mathrm{aOR}=3.6,95 \%$ CI 1 to $12.6, \mathrm{p}=0.05$ ) versus those initiating an AZT-based regimen; interestingly, different from their female counterparts, male participants showed no such association $(\mathrm{OR}=0.6,95 \% \mathrm{CI}$ 0.3 to $1.4, \mathrm{p}=0.24)$. Also, female participants had a higher risk of $\mathrm{VF}$ given adverse side effects $(\mathrm{aOR}=2.3,95 \% \mathrm{CI} 1$ to 5.6, $\mathrm{p}=0.06$ ). Male participants with missed doses in the month prior to the survey were at a higher risk of both $\mathrm{VF}$ $(\mathrm{aOR}=2.8,95 \%$ CI 1.1 to $7, \mathrm{p}=0.03)$ and HIVDR $(\mathrm{aOR}=9.7$, $95 \%$ CI 2.1 to $44.1, \mathrm{p}<0.01$ ) versus those without missed doses in the preceding month. Conversely, missed doses in prior month were not significantly associated with $\mathrm{VF}$ or HIVDR for women.

\section{HIVDR and subtype (table 4)}

HIVDR identified in our study was consistent with the NFATP-recommended ART regimen. All 27 participants detected with drug resistance had NNRTI mutations, 21 $(77.8 \%)$ had NRTI mutations. The dominant subtype was CRF07_BC for both men (61.5\%) and women (50\%). All participants found with HIVDR had developed HIVDR towards NNRTI; $85.7 \%$ of male participants and $69.2 \%$ of female participants developed HIVDR towards NRTI; no protease inhibitor mutation was detected. There were no CRF08_BC subtypes detected in the study population.

\section{DISCUSSION}

The 48-month risk of VF was $11.8 \%$ and HIVDR was $5.0 \%$, which indicated relatively good treatment outcomes given meta-analysis suggested a 37 to 48 -month HIVDR prevalence ranging from $3.04 \%$ to $47.92 \%$ in China, ${ }^{9}$ similar to studies in China which estimated a 1-year HIVDR incidence of $3.5 \%$ in 2009 and $2.1 \%$ in $2012 .{ }^{25}{ }^{26}$ Our study substantiates the finding that VF and HIVDR largely decreased since the wide spread of 3TC-based regimens. ${ }^{27}$ Studies have shown mixed findings of gender differences on ART adherence and treatment outcomes. ${ }^{14} 162829$ In our study, we found male participants had slightly higher risk of $\mathrm{VF}(12.2 \%$ vs $11.2 \%, \mathrm{p}=0.72)$ but lower risk of HIVDR ( $4.7 \%$ vs $5.4 \%, \mathrm{p}=0.69)$ than women. Drug adherence and adverse effects influenced our outcomes differently for men compared with women. Women's risks of $\mathrm{VF}$ and HIVDR were not associated with missing doses in the past month; also, few women missed doses relative to men, similar to two other studies in China suggesting women have better adherence behaviours. ${ }^{30} 31$ Male participants were at higher risk of both VF and HIVDR if they reported missed doses. More detailed studies need to be conducted on the frequency and factors associated with missing treatment. However, female participants showed a higher risk of VF if they had adverse effect while men did not. This calls for further research of what types of adverse effects are occurring and how they affect ART adherence and virological outcomes across gender.

We found in this study that women, not men, who initiated D4T-based regimens were more susceptible to $\mathrm{VF}$ (women vs men: $\mathrm{OR}=2.3,95 \%$ CI 1.0 to $5.7, \mathrm{p}=0.06$ ) and HIVDR (women vs men: $\mathrm{OR}=3.0,95 \%$ CI 0.8 to 11.3 , $\mathrm{p}=0.11$ ), consistent with previous findings that D4T was more likely to increase the risk of mitochondrial toxicity in women. ${ }^{32}{ }^{33}$ Mitochondrial toxicity caused by D4T had been reported to cause many adverse effects such as lactic acidosis, lipodystrophy and peripheral neuropathy. ${ }^{34} 35$ Following the WHO recommendation, ${ }^{36}$ the NFATP advocated switching the first-line regimen from D4T to TDF in 2012. The percentage of people living with HIV initiating D4T-based regimen changed from $34.3 \%$ in 2010 to $10 \%$ in 2012 and $0.9 \%$ in $2014^{37}$; however, there were still $29.9 \%$ of participants in our study who were on D4T-based regimens in 2012. It was noteworthy that we did not see a statistical difference in $\mathrm{VF}(\mathrm{OR}=1.4,95 \%$ CI 0.4 to $4.2, \mathrm{p}=0.60)$ and HIVDR ( $\mathrm{OR}=1.0,95 \%$ CI 0.2 to $4.2, \mathrm{p}=0.98)$ between women who initiated and remained on D4T-based regimens and those who switched to AZT/ TDF-based regimens. It is possible that women switched regimens because of VF; however, further studies need to be done to explore when to switch ART regimen for women receiving D4T-based regimens. It is important to mention that data on ART adherence and adverse effects were collected in 2012, when there were only four female participants still using D4T-based regimens who experienced VF. The sample size was not sufficient to explore whether D4T-based regimens affect drug adherence and adverse effects for women.

Though not associated with the VF and HIVDR outcomes, $38.4 \%$ of study participants reported that they would not 'always' be willing to take ART in the future, $36.8 \%$ reported not believing that ART is 'always' health 


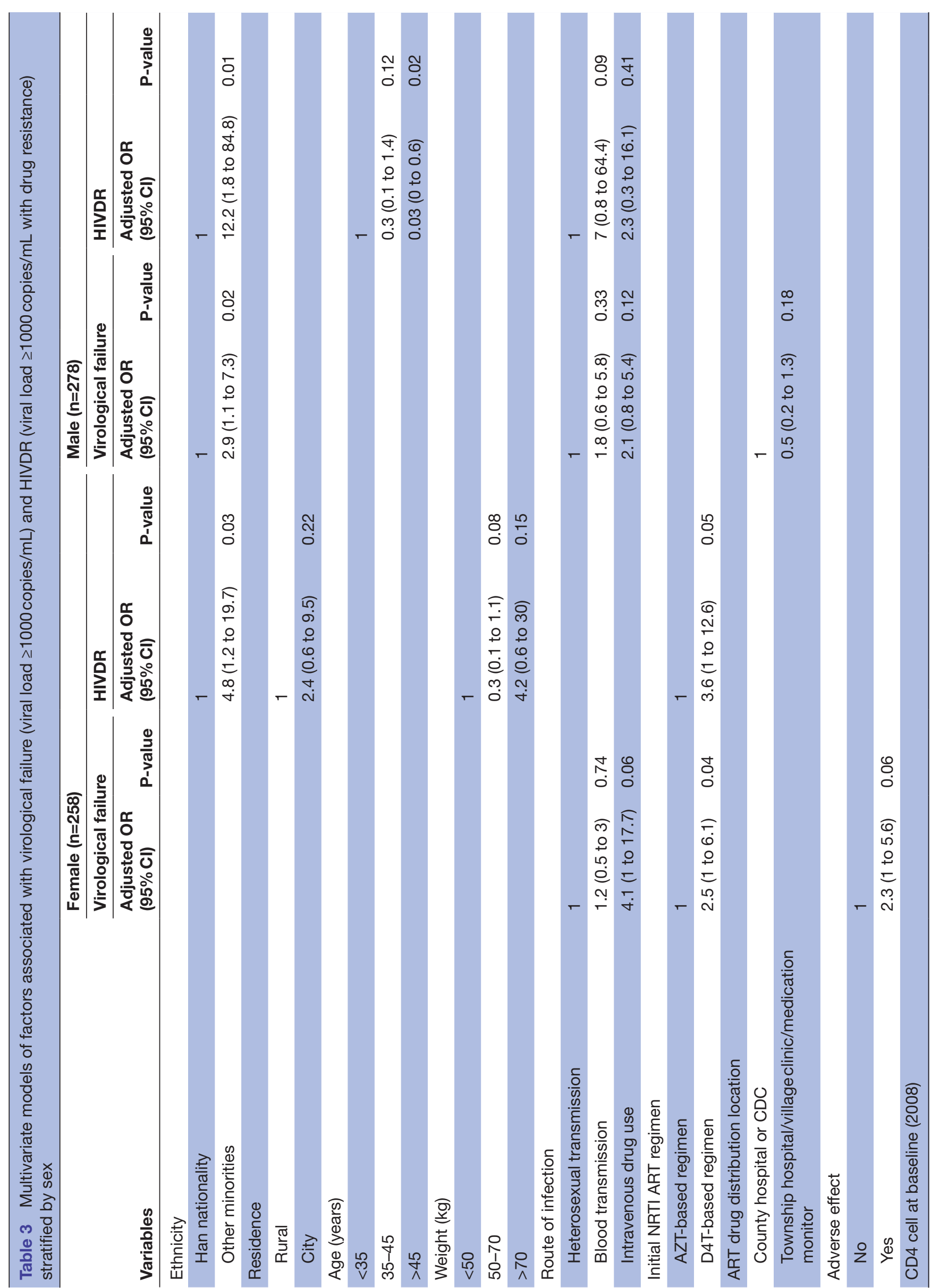

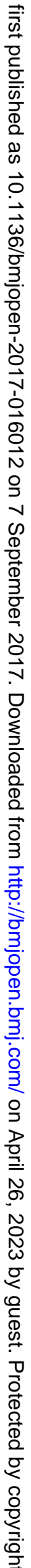




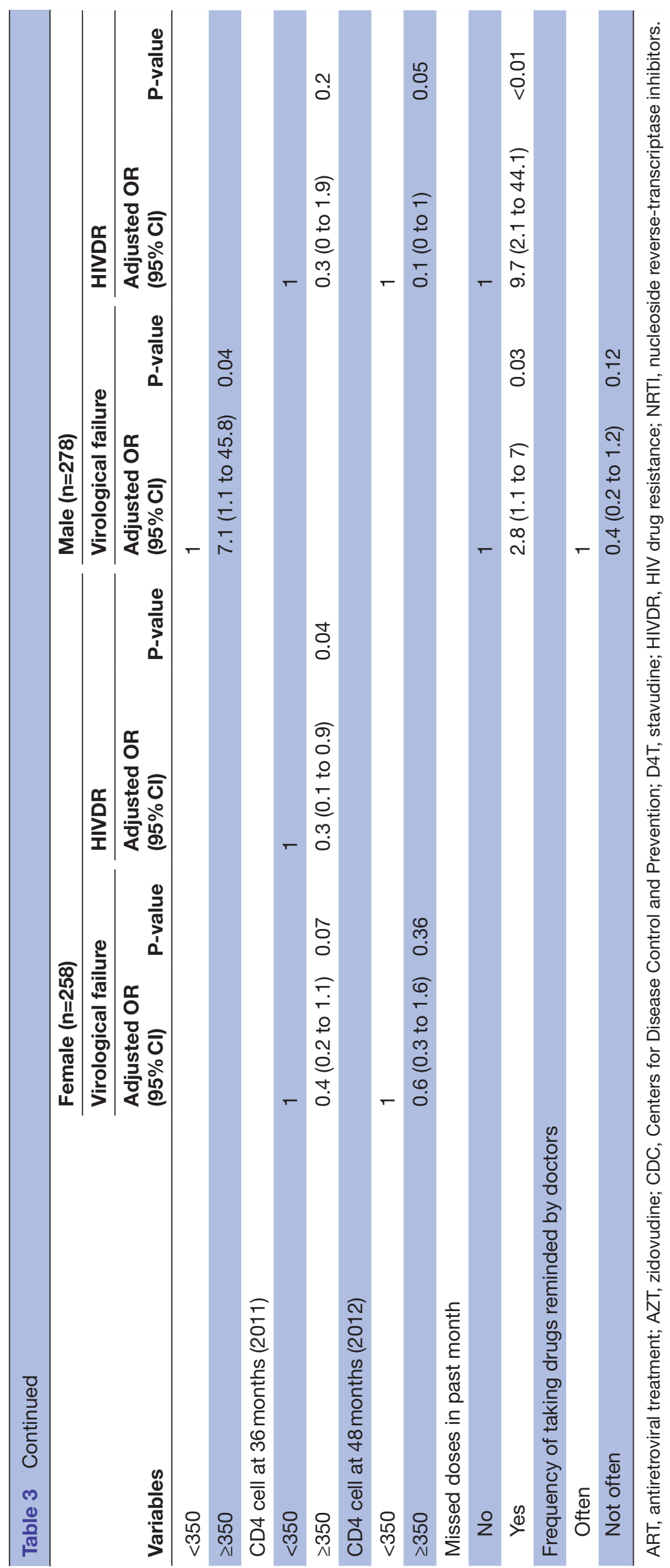

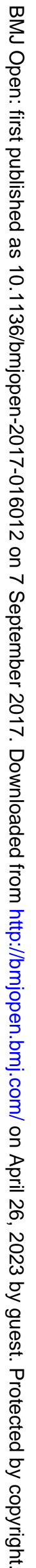


Table 4 HIVDR and subtype among 27 patients with HIVDR mutation detected at 2011 and/or 2012 stratified by sex

\begin{tabular}{|c|c|c|c|c|}
\hline & Female (\%) & Male (\%) & Mutations & N (\%) \\
\hline Overall & 14 & 13 & & \\
\hline \multicolumn{5}{|l|}{ Subtype } \\
\hline B & $5(35.7)$ & $3(23.1)$ & & \\
\hline C & & $1(7.7)$ & & \\
\hline CRF01_AE & $2(14.3)$ & $1(7.7)$ & & \\
\hline CRF07_BC & $7(50)$ & $8(61.5)$ & & \\
\hline \multicolumn{5}{|l|}{ Antiretroviral drug } \\
\hline NNRTI (any) & $14(100)$ & $13(100)$ & NNRTI mutations (total) & 27 \\
\hline Efavirenz (EFV) & $14(100)$ & $13(100)$ & V90I & $1(3.7)$ \\
\hline Nevirapine (NVP) & $14(100)$ & $13(100)$ & A98G & $2(7.4)$ \\
\hline \multirow[t]{12}{*}{ Etravirine (ETR) } & $6(42.9)$ & $8(61.5)$ & K101E & $6(22.2)$ \\
\hline & & & K103N & $11(40.7)$ \\
\hline & & & V106A & $4(14.8)$ \\
\hline & & & V108I & $6(22.2)$ \\
\hline & & & $\mathrm{E} 138 \mathrm{~A}$ & $1(3.7)$ \\
\hline & & & V179D/F & $3(11.1)$ \\
\hline & & & Y181C & $5(18.5)$ \\
\hline & & & G190A & $5(18.5)$ \\
\hline & & & $\mathrm{H} 221 \mathrm{Y}$ & $1(3.7)$ \\
\hline & & & $\mathrm{P} 225 \mathrm{H}$ & $3(11.1)$ \\
\hline & & & F227L & $1(3.7)$ \\
\hline & & & M230L & $1(3.7)$ \\
\hline NRTI (any) & $12(85.7)$ & $9(69.2)$ & NRTI mutations (total) & 21 \\
\hline Lamivudine (3TC) & $12(85.7)$ & $9(69.2)$ & A62V & $1(4.8)$ \\
\hline Azidothymidine (AZT) & $2(14.3)$ & $2(15.4)$ & D67G & $1(4.8)$ \\
\hline Tenofovir (TDF) & $1(7.1)$ & $3(23.1)$ & T69N & $2(9.5)$ \\
\hline Stavudine (D4T) & $3(21.4)$ & $3(23.1)$ & $\mathrm{K} 70 \mathrm{R} / \mathrm{Q}$ & $4(19)$ \\
\hline Didanosine (DDI) & $5(35.7)$ & $3(23.1)$ & V75I/M & $2(9.5)$ \\
\hline Abacavir (ABC) & $12(85.7)$ & $9(69.2)$ & M184V & $17(81.0)$ \\
\hline \multirow[t]{2}{*}{ Emtricitabine (FTC) } & $12(85.7)$ & $9(69.2)$ & $\mathrm{T} 215 \mathrm{~N}$ & $1(4.8)$ \\
\hline & & & K219E/Q & $2(9.5)$ \\
\hline $\mathrm{PI}$ (any) & 0 & 0 & PI mutations (total) & 0 \\
\hline
\end{tabular}

HIVDR, HIV drug resistance; NNRTI, non-nucleoside reverse-transcriptase inhibitors; NRTI, nucleoside reverse-transcriptase inhibitors; PI, protease inhibitor.

promoting, and $42.5 \%$ reported not believing that poor compliance 'always' contributed to HIVDR. As willingness and these knowledge factors may impact more longterm VF and HIVDR outcomes, the motivations behind willingness and knowledge about VF and HIVDR warrant exploration.

Caution is needed when interpreting the study results from multivariate model that older age $(>45)$ was protective for HIVDR in men. There were only four male IDU participants with older age $(>45)$ in this study, the number is not sufficient to test for interaction. There is no association between HIVDR and age $(\mathrm{OR}=6.5,95 \% \mathrm{CI}$ 1.1 to $38.1, \mathrm{p}=0.49$ ) in the subanalysis we did among male
IDU participants, after controlling for variables showed significance in the univariate model. A previous study in HIV-positive IDU population in China suggested that there is no association between $\mathrm{VF}$ and sex or age.$^{38}$ In this study, $61.5 \%$ of male participants with HIVDR became HIV infected via IDU, yet there were only $22.7 \%$ of male IDU participants.

In addition, we found that younger $(<45$ years $)$ IDU population was more likely to miss doses (18.6\%) compared with heterosexual transmission population $(8.8 \%)$ and blood transfusion transmission population $(12.1 \%)$. This finding was consistent with studies that implied younger men were at a higher risk of drug 
abuse. ${ }^{39} 40$ This result indicated that younger IDU population could be a main source of VF and HIVDR; therefore, they could be future targeted population for behavioural intervention.

The increased risk of VF and HIVDR in non-Han minorities, regardless of gender, may be due to logistical, cultural or social barriers faced by ethnic minorities which limit their adherence to ART. It has been reported that minorities tend to have lower social economic status than Han majorities, followed by lower education level and fewer access to health facilities. ${ }^{41}$ It may be difficult for health professionals to reach for some minorities because of their more remote geographical locations. Additionally, several studies have reported that the percentage of high-risk populations such as female sex workers and IDUs was higher in minorities than of Han nationality. ${ }^{42-44}$ The causes of this increased VF and HIVDR risk warrant further exploration, and ethnic minorities may be an important group to tailor adherence-focused interventions in China. The finding that higher CD4 cell count at follow-up was protective for VF and HIVDR was expected. NFATP changed treatment criteria from CD4 cell count $\leq 0.2 \times 10^{\wedge} 9 / \mathrm{L}$ to $\mathrm{CD} 4$ cell count $\leq 0.3 \times 10^{\wedge} 9 / \mathrm{L}$ following the WHO recommendation in 2011. ${ }^{7175}$ Our study indicated that male participants who initiated treatment in 2008 at $\mathrm{CD} 4$ cell count $\geq 0.35 \times 10^{\wedge} 9 / \mathrm{L}$ were still at higher risk towards VF (aOR=7.1, 95\% CI 1.1 to 45.8 , $\mathrm{p}=0.04$ ), supporting possible clinical benefits of initiating ART at higher CD4 cell counts $<0.5 \times 10^{\wedge} 9 / \mathrm{L}$ as per $\mathrm{WHO}$ recommendation in $2013 .{ }^{46}$

Among participants infected by blood transmission, we only found HIVDR subtype B; only one subtype $\mathrm{C}$ was found in participants infected though IDU, the dominant subtype was CRF07_BC, found both in participants infected by heterosexual transmission and IDU. The most common NNRTI mutation sites were K103N (40.7\%), K101E (22.2\%) and V108I (22.2\%); the most common NRTI mutation sites were M184V (81.0\%) and K70R (19\%). Interestingly, compared with a 1-year follow-up study in China with all participants initiated ART in $2011,{ }^{47}$ there is no V108I in their study and we did not find $\mathrm{K} 65 \mathrm{R}$ in our study.

Study findings should be interpreted in light of several limitations. Though we did not account for transmitted drug resistance in this study, previous studies have found low transmitted drug resistance risk $(<5 \%)$ during this period $^{48} 4950$; we could be fairly certain that participants were outcome free in 2008 as they were new ART initiators. Another limitation of our study is that the outcomes were measured in 2011 and 2012, and thus we may be missing transient VF outcomes. Also, route of transmission was collected in 2008 when assessing HIV infection among men who have sex with men was not part of data collection instruments; additionally, we do not have data on sex worker status. Roughly half of the study participants selected for possible inclusion in the study for having initiated first-line ART in 2008 were lost to follow-up by 2012, creating a possible selection bias for individuals with better ART adherence-this bias may underestimate the true VF and HIVDR risk and also limit the generalisability of our findings to better adherers. Additionally, given the demographic profile of the cohort, our findings are most generalisable to heterosexual Han nationals who are married/cohabiting and working as agricultural labourers in rural areas. Misclassification of self-reported data is possible, though we do not expect this misclassification to be differential by the outcome of interest and thus any such information bias would bias our results towards the null.

\section{CONCLUSIONS}

We found female participants initiating D4T versus AZT-based regimens were more vulnerable to $\mathrm{VF}$ and HIVDR, and we suggest future studies on whether and when to change ART regimen for women initiated with D4T-based regimen. Poor adherence was a risk factor among male participants who may benefit from reinforced adherence counselling or social support. Increased VF and HIVDR risk among non-Han minorities warrants further exploration, and ethnic minorities may be an important group to tailor adherence-focused interventions in China. Also, this study indicated that younger men who become infected through intravenous drug use may be groups to strategically focus counselling and increasing adherence support programme.

\section{Author affiliations}

${ }^{1}$ Division of Virology and Immunology, State Key Laboratory for Infectious Disease Prevention and Control, National Center for AIDS/STD Control and Prevention, Chinese Center for Disease Control and Prevention, Collaborative Innovation Center for Diagnosis and Treatment of Infectious Diseases, Beijing, China

${ }^{2}$ Department of HIV/AIDS Control and Prevention, Guangxi Center for Disease Control and Prevention, Nanning, China

${ }^{3}$ Department of HIV/AIDS Control and Prevention, Yunnan Center for Disease Control and Prevention, Kunming, China

${ }^{4}$ Department of HIV/AIDS Control and Prevention, Hubei Center for Disease Control and Prevention, Kunming, China

${ }^{5}$ Department of HIV/AIDS Control and Prevention, Xinjiang Autonomous Region Center for Disease Control and Prevention, Urumqi, China

${ }^{6}$ Department of HIV/AIDS Control and Prevention, Henan Center for Disease Control and Prevention, Zhengzhou, China

${ }^{7}$ Department of Epidemiology, Rollins School of Public Health, Emory University, Atlanta, Georgia, USA

${ }^{8}$ Department of Global Health, Rollins School of Public Health, Emory University, Atlanta, Georgia, USA

${ }^{9}$ Division of Infectious Diseases, Emory University School of Medicine, Atlanta, Georgia, USA

${ }^{10}$ Division of Virology and Immunology, Chinese Center for AIDS/STD Control and Prevention, Beijing, China

Contributors YR, WK, HX and YS designed the study. YR, HX, SL, LL, YM, HT, TZ, GS, $\mathrm{CH}, \mathrm{WK}, \mathrm{XL}$ and $\mathrm{PL}$ collected the data. $\mathrm{CH}, \mathrm{TT}$ and $\mathrm{LL}$ conducted laboratory analysis. $\mathrm{XL}$ and WK analysed the data. KMW, VCM, YR, WK, HX, LL and YS interpreted the data. KMW, VCM, YR, WK, TT and YS drafted the report. All authors reviewed, revised and approved the final report.

Funding This study was supported by Guangxi Center for Disease Control and Prevention, Nanning, China. The antiretroviral drugs used in this study were provided by NFATP.

Competing interests None declared.

Patient consent Obtained. 
Ethics approval National Center for AIDS/STD Control and Prevention of the Chinese Center for Disease Control and Prevention (NCAIDS, China CDC).

Provenance and peer review Not commissioned; externally peer reviewed.

Data sharing statement № additional data are available.

Open Access This is an Open Access article distributed in accordance with the Creative Commons Attribution Non Commercial (CC BY-NC 4.0) license, which permits others to distribute, remix, adapt, build upon this work non-commercially, and license their derivative works on different terms, provided the original work is properly cited and the use is non-commercial. See: http://creativecommons.org/ licenses/by-nc/4.0/

(C) Article author(s) (or their employer(s) unless otherwise stated in the text of the article) 2017. All rights reserved. No commercial use is permitted unless otherwise expressly granted.

\section{REFERENCES}

1. Hong SY, Nachega JB, Kelley K, et al. The global status of HIV drug resistance: clinical and public-health approaches for detection, treatment and prevention. Infect Disord Drug Targets 2011;11:124-33.

2. Gabillard D, Lewden C, Ndoye I, et al. Mortality, AIDS-morbidity, and loss to follow-up by current CD4 cell count among HIV-1-infected adults receiving antiretroviral therapy in Africa and Asia: data from the ANRS 12222 collaboration. J Acquir Immune Defic Syndr 2013:62:555-61.

3. Montaner JS, Lima VD, Harrigan PR, et al. Expansion of HAART coverage is associated with sustained decreases in HIV/AIDS morbidity, mortality and HIV transmission: the "HIV Treatment as Prevention" experience in a Canadian setting. PLoS One 2014;9:e87872.

4. Staszewski S, Morales-Ramirez J, Tashima KT, et al. Efavirenz plus zidovudine and lamivudine, efavirenz plus indinavir, and indinavir plus zidovudine and lamivudine in the treatment of HIV-1 infection in adults. Study 006 Team. N Engl J Med 1999;341:1865-73.

5. Wang X, Yang L, Li H, et al. Factors associated with HIV virologic failure among patients on HAART for one year at three sentinel surveillance sites in China. Curr HIV Res 2011;9:103-11.

6. Zhang F, Dou Z, Ma Y, et al. Five-year outcomes of the China National Free Antiretroviral Treatment Program. Ann Intern Med 2009;151:241-51.

7. Chinese Center of Disease and Control. Manual of the national free antiretroviral treatment, 2007

8. People's Republic of China (2008). UNGASS country progress report. Beijing: People's Republic of China, 2007.

9. Liu H, Ma Y, Su Y, et al. Emerging trends of HIV drug resistance in Chinese HIV-infected patients receiving first-line highly active antiretroviral therapy: a systematic review and meta-analysis. Clin Infect Dis 2014;59:1495-502.

10. Djarma $O$, Nguyen $Y$, Renois $F$, et al. Continuous free access to HAART could be one of the potential factors impacting on loss to follow-up in HAART-eligible patients living in a resourcelimited setting: N'djamena, Chad. Trans $R$ Soc Trop Med Hyg 2014;108:735-8.

11. DeGruttola V, Dix L, D'Aquila R, et al. The relation between baseline HIV drug resistance and response to antiretroviral therapy: re-analysis of retrospective and prospective studies using a standardized data analysis plan. Antivir Ther 2000;5:41-8.

12. Liao L, Xing H, Su B, et al. Impact of HIV drug resistance on virologic and immunologic failure and mortality in a cohort of patients on antiretroviral therapy in China. AIDS 2013;27:1815-24.

13. Hare AQ, Ordóñez CE, Johnson BA, et al. Gender-specific risk factors for virologic failure in KwaZulu-Natal: automobile ownership and financial insecurity. AIDS Behav 2014;18:2219-29.

14. Berg KM, Demas PA, Howard AA, et al. Gender differences in factors associated with adherence to antiretroviral therapy. J Gen Intern Med 2004;19:1111-7.

15. Floridia M, Giuliano M, Palmisano L, et al. Gender differences in the treatment of HIV infection. Pharmacol Res 2008;58:173-82.

16. Moore AL, Mocroft A, Madge S, et al. Gender differences in virologic response to treatment in an HIV-positive population: a cohort study. J Acquir Immune Defic Syndr 2001;26:159-63.

17. World Health Organization. Surveillance of HIV drug resistance In adults receiving art (acquired Hiv drug resistance). 2014 (accessed Jul 2014).

18. Bennett DE, Myatt M, Bertagnolio S, et al. Recommendations for surveillance of transmitted HIV drug resistance in countries scaling up antiretroviral treatment. Antivir Ther 2008;13(Suppl 2):25-36.
19. Gaydos CA, Rizzo-Price PA, Balakrishnan P, et al. Impact of international laboratory partnerships on the performance of HIV/ sexually transmitted infection testing in five resource-constrained countries. Int J STD AIDS 2011;22:645-52.

20. World Health Organization. World health organization protocol for population based monitoring of HIV drug resistance emerging during treatment and related program factors at sentinel antiretroviral therapy clinics.

21. Jiang Y, Qiu M, Zhang G, et al. Quality assurance in the HIV/ AIDS laboratory network of China. Int J Epidemiol 2010;39(Suppl 2):ii72-ii78.

22. Liu TF, Shafer RW. Web resources for HIV type 1 genotypicresistance test interpretation. Clin Infect Dis 2006;42:1608-18.

23. Yan $\mathrm{H}, \mathrm{Yu} \mathrm{H}$, Xing W, et al. Development of a proficiency testing program for the HIV-1 BED incidence assay in China. Sci Rep 2014;4:4512.

24. Zhong P, Pan Q, Ning Z, et al. Genetic diversity and drug resistance of human immunodeficiency virus type 1 (HIV-1) strains circulating in Shanghai. AIDS Res Hum Retroviruses 2007;23:847-56.

25. Wang $\mathrm{J}, \mathrm{He} \mathrm{C}$, $\mathrm{Hsi} \mathrm{JH}$, et al. Virological outcomes and drug resistance in Chinese patients after 12 months of 3TC-based firstline antiretroviral treatment, 2011-2012. PLoS One 2014;9:e88305.

26. Xing $\mathrm{H}$, Ruan $\mathrm{Y}, \mathrm{Hsi} \mathrm{JH}$, et al. Reductions in virological failure and drug resistance in Chinese antiretroviral-treated patients due to lamivudine-based regimens, 2003-12. J Antimicrob Chemother 2015;70:dkv078.

27. Nicastri $\mathrm{E}$, Leone $\mathrm{S}$, Angeletti $\mathrm{C}$, et al. Sex issues in HIV-1-infected persons during highly active antiretroviral therapy: a systematic review. J Antimicrob Chemother 2007;60:724-32.

28. Puskas CM, Forrest JI, Parashar S, et al. Women and vulnerability to HAART non-adherence: a literature review of treatment adherence by gender from 2000 to 2011. Curr HIVIAIDS Rep 2011;8:277-87.

29. Dou Z, Xu J, Jiao JH, et al. Gender difference in 2-year mortality and immunological response to ART in an HIV-infected Chinese population, 2006-2008. PLoS One 2011;6:e22707.

30. Sabin LL, Desilva MB, Hamer DH, et al. Barriers to adherence to antiretroviral medications among patients living with HIV in southern China: a qualitative study. AIDS Care 2008;20:1242-50.

31. Wester CW, Okezie OA, Thomas AM, et al. Higher-than-expected rates of lactic acidosis among highly active antiretroviral therapy-treated women in Botswana: preliminary results from a large randomized clinical trial. J Acquir Immune Defic Syndr 2007;46:318-22

32. Goedecke JH, Micklesfield LK, Levitt NS, et al. Effect of different antiretroviral drug regimens on body fat distribution of HIV-infected South African women. AIDS Res Hum Retroviruses 2013;29:557-63.

33. Feleke Y, Fekade D, Mezegebu Y. Prevalence of highly active antiretroviral therapy associated metabolic abnormalities and lipodystrophy in HIV infected patients. Ethiop Med J 2012;50:221-30.

34. Moyle GJ, Nelson MR, Hawkins D, et al. The use and toxicity of didanosine (ddl) in HIV antibody-positive individuals intolerant to zidovudine (AZT). Q J Med 1993;86:155-63.

35. Geneva. Consolidated guidelines on the use of antiretroviral drugs for treating and preventing HIV infection: recommendations for a public health approach, 2013.

36. Dou Z, Zhang F, Zhao Y, et al. [Progress on China' s national free antiretroviral therapy strategy in 2002-2014]. Zhonghua Liu Xing Bing Xue Za Zhi 2015;36:1345-50.

37. Leng $X$, Liang $S, M a Y$, et al. HIV virological failure and drug resistance among injecting drug users receiving first-line ART in China. BMJ Open 2014:4:e005886.

38. Wu Z, Zhang J, Detels R, et al. Characteristics of risk-taking behaviors, HIV and AIDS knowledge, and risk perception among young males in southwest China. AIDS Educ Prev 1997;9:147-60.

39. Yang $\mathrm{H}, \mathrm{Li} X$, Stanton $\mathrm{B}$, et al. Heterosexual transmission of HIV in China: a systematic review of behavioral studies in the past two decades. Sex Transm Dis 2005;32:270-80.

40. Myers SL, Xiaoyan G, Cruz BC. Ethnic minorities, race, and inequality in China: A new perspective on racial dynamics. Rev Black Polit Econ 2013;40:231-44.

41. Zoufaly A, Jochum J, Hammerl R, et al. Virological failure after 1 year of first-line ART is not associated with HIV minority drug resistance in rural Cameroon. J Antimicrob Chemother 2015;70:922-5.

42. Liu S, Wang QX, Nan L, et al. The changing trends of HIV/AIDS in an ethnic minority region of China: modeling the epidemic in Liangshan prefecture, Sichuan Province. Biomed Environ Sci 2013;26:562-70.

43. Zhang L, Zhu J, Rui B, et al. High HIV risk among Uigur minority ethnic drug users in northwestern China. Trop Med Int Health 2008;13:814-7. 
44. Chinese Center for Disease Control and Prevention. Manual of the national free antiretroviral treatment, third edition. (accessed 12 Jun 2013).

45. World Health Organization. Consolidated guidelines on general HIV care and the use of antiretroviral drugs for treating and preventing HIV infection: recommendations for a public health approach: World Health Organization, 2013

46. Xing $\mathrm{H}$, Ruan $\mathrm{Y}, \mathrm{Hsi} \mathrm{JH}$, et al. Reductions in virological failure and drug resistance in Chinese antiretroviral-treated patients due to lamivudine-based regimens, 2003-12. J Antimicrob Chemother 2015;70:2097-103.
47. Liao L, Xing $\mathrm{H}$, Shang $\mathrm{H}$, et al. The prevalence of transmitted antiretroviral drug resistance in treatment-naive HIV-infected individuals in China. J Acquir Immune Defic Syndr 2010;53(Suppl 1):S10-14.

48. Zhao K, Kang W, Liu Q, et al. Genotypes and transmitted drug resistance among treatment-naive HIV-1-infected patients in a northwestern province, China: trends from 2003 to 2013. PLoS One 2014;9:e109821.

49. Su Y, Zhang F, Liu H, et al. The prevalence of HIV-1 drug resistance among antiretroviral treatment naïve individuals in mainland China: a meta-analysis. PLOS One 2014;9:e110652.

50. Xing $\mathrm{H}$, Wang $\mathrm{X}$, Liao $\mathrm{L}$, et al. Incidence and associated factors of HIV drug resistance in Chinese HIV-infected patients receiving antiretroviral treatment. PLoS One 2013;8:e62408. 